\title{
3DeepM: An Ad Hoc Architecture Based on Deep Learning Methods for Multispectral Image Classification
}

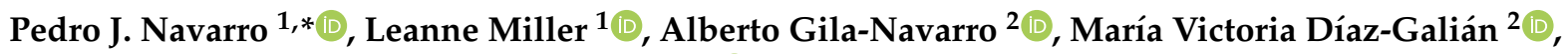 \\ Diego J. Aguila ${ }^{3}$ and Marcos Egea-Cortines ${ }^{2}$ D \\ 1 Escuela Técnica Superior de Ingeniería de Telecomunicación (DSIE), Campus Muralla del Mar, s/n, \\ Universidad Politécnica de Cartagena, 30202 Cartagena, Spain; leanne.miller@upct.es \\ 2 Genética Molecular, Instituto de Biotecnología Vegetal, Edificio I+D+I, Plaza del Hospital s/n, \\ Universidad Politécnica de Cartagena, 30202 Cartagena, Spain; alberto.gilan@um.es (A.G.-N.); \\ mariavictoria.diaz@edu.upct.es (M.V.D.-G.); marcos.egea@upct.es (M.E.-C.) \\ 3 Sociedad Cooperativa Las Cabezuelas, 30840 Alhama de Murcia, Spain; diego@lascabezuelas.com \\ * Correspondence: pedroj.navarro@upct.es; Tel.: +34-968326546
}

Citation: Navarro, P.J.; Miller, L.; Gila-Navarro, A.; Díaz-Galián, M.V.; Aguila, D.J.; Egea-Cortines, M. 3DeepM: An Ad Hoc Architecture Based on Deep Learning Methods for Multispectral Image Classification. Remote Sens. 2021, 13, 729. https:// doi.org/10.3390/rs13040729

Academic Editor: Qi Wang

Received: 17 January 2021

Accepted: 11 February 2021

Published: 17 February 2021

Publisher's Note: MDPI stays neutral with regard to jurisdictional claims in published maps and institutional affiliations.

Copyright: (c) 2021 by the authors. Licensee MDPI, Basel, Switzerland. This article is an open access article distributed under the terms and conditions of the Creative Commons Attribution (CC BY) license (https:/ / creativecommons.org/licenses/by/ $4.0 /)$.

\begin{abstract}
Current predefined architectures for deep learning are computationally very heavy and use tens of millions of parameters. Thus, computational costs may be prohibitive for many experimental or technological setups. We developed an ad hoc architecture for the classification of multispectral images using deep learning techniques. The architecture, called 3DeepM, is composed of 3D filter banks especially designed for the extraction of spatial-spectral features in multichannel images. The new architecture has been tested on a sample of 12210 multispectral images of seedless table grape varieties: Autumn Royal, Crimson Seedless, Itum4, Itum5 and Itum9. 3DeepM was able to classify 100\% of the images and obtained the best overall results in terms of accuracy, number of classes, number of parameters and training time compared to similar work. In addition, this paper presents a flexible and reconfigurable computer vision system designed for the acquisition of multispectral images in the range of $400 \mathrm{~nm}$ to $1000 \mathrm{~nm}$. The vision system enabled the creation of the first dataset consisting of 12210 37-channel multispectral images (12 VIS + 25 IR) of five seedless table grape varieties that have been used to validate the 3DeepM architecture. Compared to predefined classification architectures such as AlexNet, ResNet or ad hoc architectures with a very high number of parameters, 3DeepM shows the best classification performance despite using 130-fold fewer parameters than the architecture to which it was compared. 3DeepM can be used in a multitude of applications that use multispectral images, such as remote sensing or medical diagnosis. In addition, the small number of parameters of 3DeepM make it ideal for application in online classification systems aboard autonomous robots or unmanned vehicles.
\end{abstract}

Keywords: deep learning architectures; multispectral grape classification; multispectral computer vision system

\section{Introduction}

Computer vision and spectral imaging techniques are becoming increasingly popular in the agricultural and food industries for performing tasks such as classification and quality control. As consumers are demanding higher quality of food products at a reasonable price, producers are faced with the challenge of performing the tasks of classification and inspection more efficiently and rapidly. These tasks have traditionally been performed manually, which is usually a slow and costly process and depends on the features to be detected being visible to the human eye, which is often not the case [1].

Deep learning has become a valuable tool when it comes to classification and quality control in the agricultural industry due to its powerful and fast image feature extraction. An interesting study was performed by Pereira et al. in [2] for the classification of grape varieties using their own RGB images of bunches of grapes taken in vineyards. Due to the 
small number of samples obtained, they used data augmentation to create fake samples, increasing the size of the dataset. A pretrained AlexNet architecture was used to classify six different grape varieties, and an accuracy of $77.30 \%$ was achieved.

In the work performed by [3], with a dataset of RGB images containing 408 bunches of wine grapes of five different varieties in a vineyard. Mask R-CNN and YOLO networks were trained, with the best results obtained by the Mask R-CNN network, which achieved a confidence level of 0.91 . A CNN architecture was designed in [4] to classify grapes according to their colour, and a Kaggle open dataset containing 4565 RGB images of individual grape grains was used. An accuracy of $100 \%$ was obtained, although the task of simply identifying grape colour is not a complex one.

Deep learning methods are being used not only for the close-range classification of crops, but there is also a growing interest in their use for crop identification and landuse classification using multispectral satellite images [5]. Various CNN models for crop identification have been designed, trained and tested using hyperspectral remote sensing images [6]. In [7], an accuracy of 97.58 was obtained using the Indian Pines dataset with 16 different classes. In [8], six different $\mathrm{CNN}$ architectures for crop classification were trained using multispectral land cover maps containing 14 classes, with the best $\mathrm{CNN}$ model obtaining an overall accuracy of $78-85 \%$ with the test samples.

Hyperspectral imaging techniques have also been used in a variety of studies in recent years, such as classification and sorting [9]; quality assessment of food products, for example, meat [10] or fruit [11]; disease detection in plants and crops [12,13]; and aerial scene classification [14], to name a few.

Hyperspectral cameras capture images for different wavelengths in the electromagnetic spectrum, from $400 \mathrm{~nm}$ (visible light) to $1300 \mathrm{~nm}$ (Near Infrared, NIR) [15]. These cameras measure the amount of light emitted by an object, which is known as spectral reflectance. A series of images of the object are obtained, which represent the spectral signature obtained by the reflectance measurements of the object at different wavelength channels [16].

An early study using hyperspectral imaging was performed in 2001 by Lacar et al. for the mapping of grape varieties in a vineyard [17]. The authors used statistical analysis to compare the two grape varieties and showed that spectral differences existed in the visible region $(400-700 \mathrm{~nm})$. This study demonstrated that it would be possible to map grape varieties in a vineyard using hyperspectral imaging techniques.

More recently, a GoogleNet architecture pretrained with RGB images was used by [9] to classify hyperspectral images of 13 different types of fruit in staged scenes. With a limited dataset, the authors were able to obtain an accuracy of $92.23 \%$. Hyperspectral imaging allows the assessment of plum quality attributes, such as colour, firmness and soluble solid content (SSC), for two varieties of plum [11]. A partial least squares regression model gives good correlations for colour and the SSC; however the results for firmness prediction are less accurate.

To classify powdery mildew infection levels in grapes, Knauer et al. used hyperspectral images of detached wine grape berries [18]. A modified random forest classifier was used, and an accuracy of up to $99.8 \%$ was achieved for classifying grape berries as healthy, infected or severely diseased. In the work performed by [19], hyperspectral images of blueberries were used to train different $\mathrm{CNN}$ architectures. The $\mathrm{CNN}$ developed obtained a performance $96.96 \%$ for the classification of blueberries according to their level of freshness or decay. Spatial and spectral features were extracted from complete hyperspectral images, and the proposed network improved on the results obtained with AlexNet and GoogleNet with the same images.

GoogleNet, Alexnet, Retnet and YOLO architectures, among others, have been designed for the classification of thousands of objects, and their hundreds of hyperparameters have been adjusted through long and costly computer processes with millions of training images. The reuse of these architectures in classification problems reduces the design time of classifiers and avoids the tedious processes of adjusting network hyperparameters and 
training. From an implementation point of view, these architectures are made up of tens of millions of parameters that must be loaded into memory for use in a final application. This fact implies a high computational cost and forces the use of dedicated systems based on GPUs for their final implementation.

In this work, we present a new ad hoc architecture consisting of 3D filter banks for the extraction of features in multispectral images. The architecture has been tested for the classification of multispectral images of seedless grape varieties. The results have shown that the developed architecture is lighter and has a better performance than the classification works with which it was compared.

\section{Materials and Methods}

To be effective, deep learning techniques require large volumes of data, which can be obtained from datasets published by the research community $[20,21]$ or can be tailored to the needs of the project. In this work and continuing previous published works [22,23], a new flexible and reconfigurable multispectral computer vision system has been created for the capture of large volumes of multispectral images (MSIs). Figure 1 presents the flow diagram for the acquisition, pre-processing and data augmentation processes.
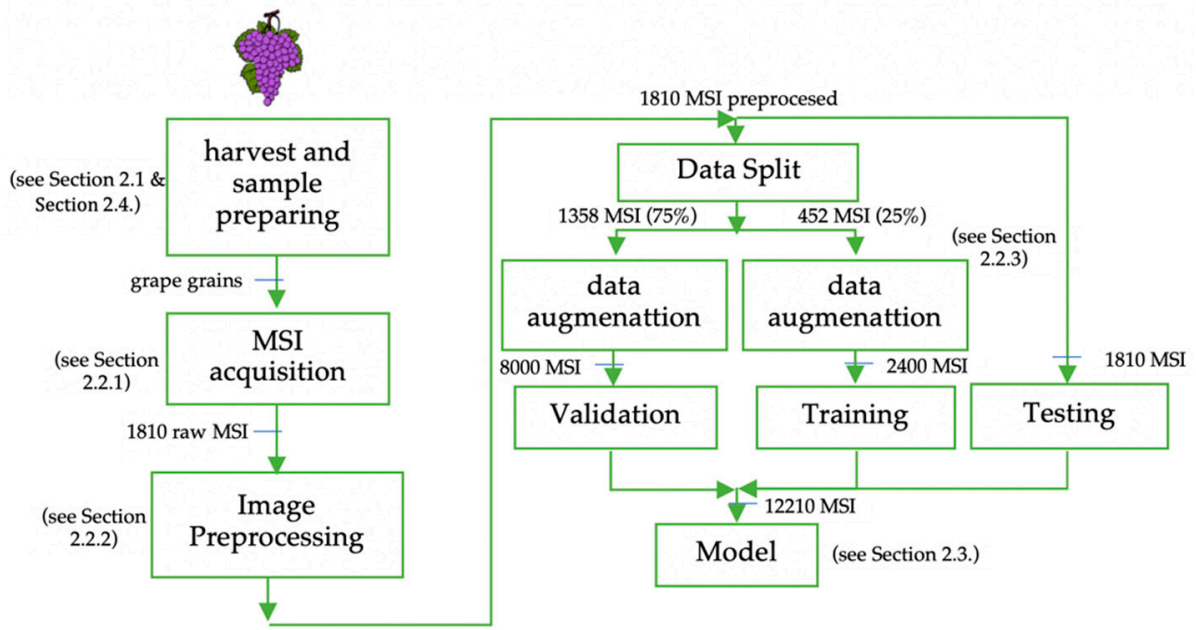

Figure 1. Flow diagram for the acquisition and data augmentation processes.

\subsection{Multispectral Computer Vision System}

The multispectral computer vision system is composed of (1) a dark chamber, (2) an illumination subsystem, (3) a multispectral image capturing subsystem and (4) a processing subsystem.

\subsubsection{Dark Chamber}

The dark chamber has a dimension of $1000 \times 1000 \times 500 \mathrm{~mm}^{3}$ and has been designed to carry out MSI captures of biological systems of different sizes, such as plant organs (flowers, stems or leaves), fruit, vegetables and so on. Depending on the type of object to analyse, the chamber can be configured to avoid undesired reflections, occlusions or shadows. For this reason, it possesses mobile panels, position accessories for cameras and illumination and elements made with a 3D printer, such as domes and semidomes.

Figure 2 shows four configurations of the dark chamber for different experiments: Figure 2a shows direct illumination to capture an MSI for growth analysis of petunia leaves [24]; Figure $2 b$ allows images to be captured with direct illumination with a dome to avoid the reflection of the illumination subsystem on the grape skin and a semidome to eliminate shadows; Figure $2 c$ shows a direct illumination configuration for the simultaneous visible and IR image capturing, avoiding unwanted brightness; and Figure $2 \mathrm{~d}$ shows a configuration with back-illumination for the simultaneous visible and IR imaging capture with a big dome to allow for correct light distribution. 


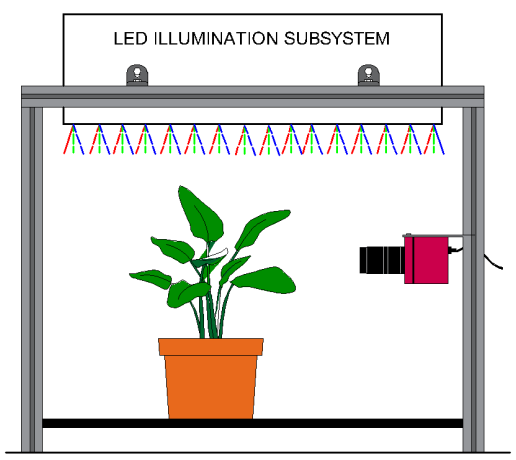

(a)

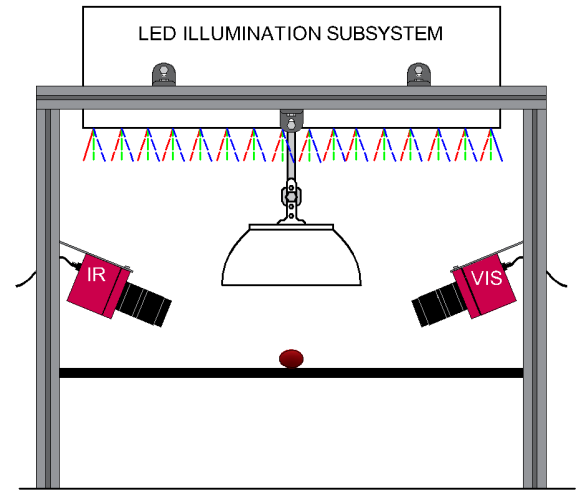

(c)

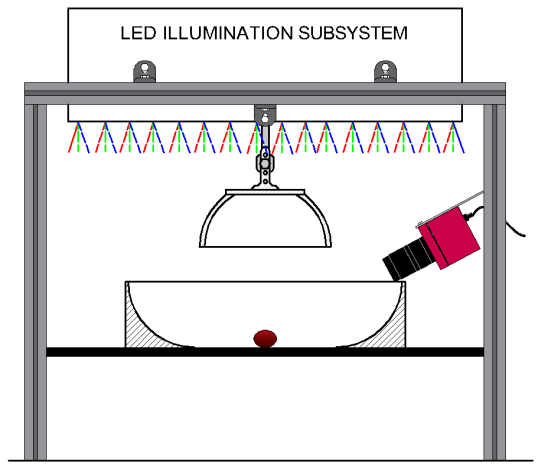

(b)

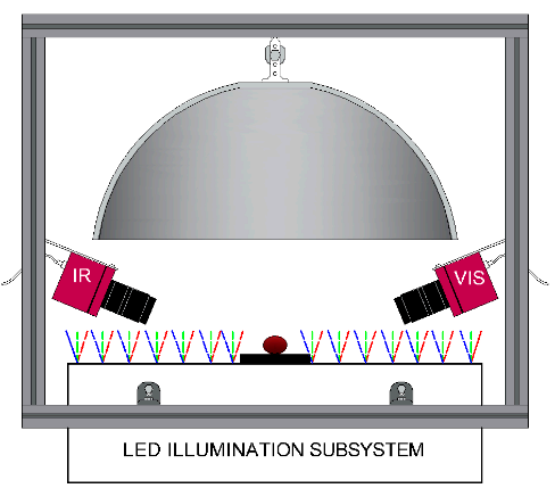

(d)

Figure 2. Flexible and reconfigurable dark chamber: (a) multispectral image (MSI) capture for growth analysis in plants, (b) configuration for suppression of unwanted reflexion and shadows from the illumination subsystem, (c) simultaneous visible and IR image acquisition with direct illumination and (d) back-illumination for the simultaneous visible and IR imaging capture.

\subsubsection{Illumination Subsystem}

The illumination subsystem is formed of an electronic module based on a microcontroller capable of managing up to 12 output channels. The module supplies the necessary power to an array of LEDs of different wavelengths, as shown in Figure 3. The power of each channel is configured by means of the lighting control panel of the MSI acquisition subsystem.

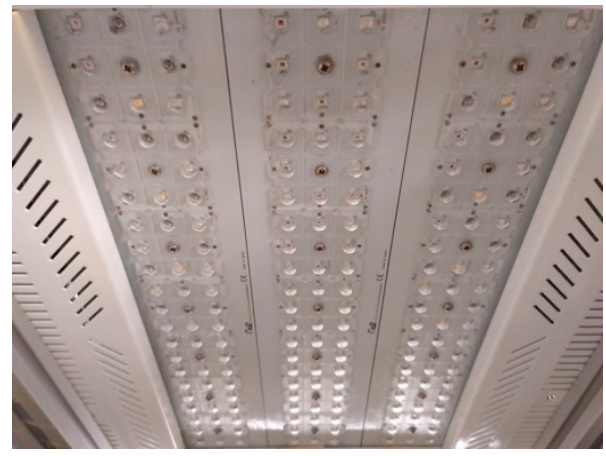

(a)

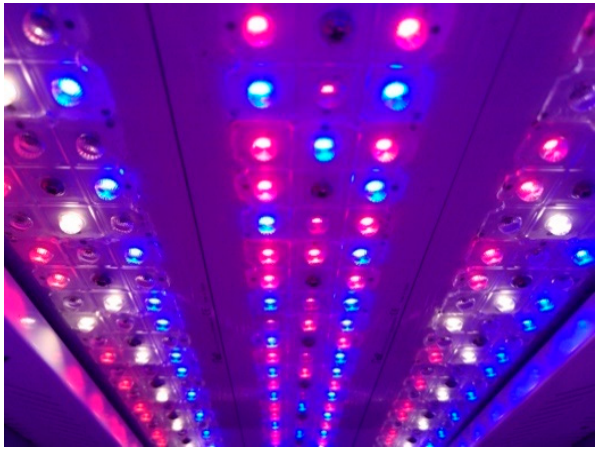

(b)

Figure 3. Illumination subsystem composed of LED arrays of different wavelengths: (a) OFF, (b) ON.

The design of the illumination subsystem allows you to add or change the LEDs connected to each channel, as long as they do not exceed the output power of the chan- 
nel. Table 1 shows the channel number, the spectra and the power per channel of the illumination subsystem.

Table 1. Channels, spectral range and power per channel.

\begin{tabular}{cccccccc}
\hline Channel & C1 & C2 & C3 & C4 & C5 & C6 & C7 \\
\hline Spectra & White & $450 \mathrm{~nm}$ & $465 \mathrm{~nm}$ & $630 \mathrm{~nm}$ & $660 \mathrm{~nm}$ & $730 \mathrm{~nm}$ & $\mathrm{IR}^{1}$ \\
Power & $24 \mathrm{~W}$ & $24 \mathrm{~W}$ & $24 \mathrm{~W}$ & $24 \mathrm{~W}$ & $24 \mathrm{~W}$ & $24 \mathrm{~W}$ & $100 \mathrm{~W}$ \\
\hline
\end{tabular}

${ }^{1}$ IR LEDS (760-800-820-840-880-910-940-970) nm.

\subsubsection{MSI Acquisition Subsystem}

The main elements of the subsystem consist of two multispectral cameras of mosaic type from Photonfocus: MV1-D2048x1088-HS03-96-G2 [25] and MV1-D2048x1088-HS0296-G2 [26]. The first camera (HS03) has 16 band-pass filters in the spectral range of $480 \mathrm{~nm}-630 \mathrm{~nm}$, while the second camera (HS02) has 25 band-pass filters in the spectral range of $600 \mathrm{~nm}-995 \mathrm{~nm}$. Both cameras can capture up to 41 raw images of 1 byte per pixel in the spectral range of $480 \mathrm{~nm}-995 \mathrm{~nm}$. After each image capture, a calibration process with two reference images to correct the reflectance values is necessary, as shown in Equation (1):

$$
I_{\text {cal }}=\frac{I_{\text {raw }}\left(t_{1}\right)-I_{\text {dark }}\left(t_{1}\right)}{I_{\text {white }}\left(t_{2}\right)-I_{\text {dark }}\left(t_{2}\right)}
$$

where $I_{\text {raw }}$ and $I_{\text {white }}$ are two multispectral images captured with a specific configuration of the illumination subsystem over the object to study and over a white reference surface, respectively; $I_{\text {dark }}$ is a multispectral image captured over a black surface in the same illumination conditions; and finally, $t_{1}$ and $t_{2}$ refer to two different exposure times used for capturing the image.

To manage and control the MSI acquisition subsystem, a friendly and easy-to-use user interface programmed using LabVIEW on a host computer has been developed, which allows (see Figure 4) one (1) to set the parameters of the multispectral cameras and to obtain calibrated images; (2) to configure the output power of the illumination subsystem (for that, a UDP communication protocol between the host computer and illumination subsystem has been developed); (3) to carry out experiments based on a temporal schedule triggering the multispectral cameras and illumination subsystem; and (4) to log the events of the experiment and handle errors.

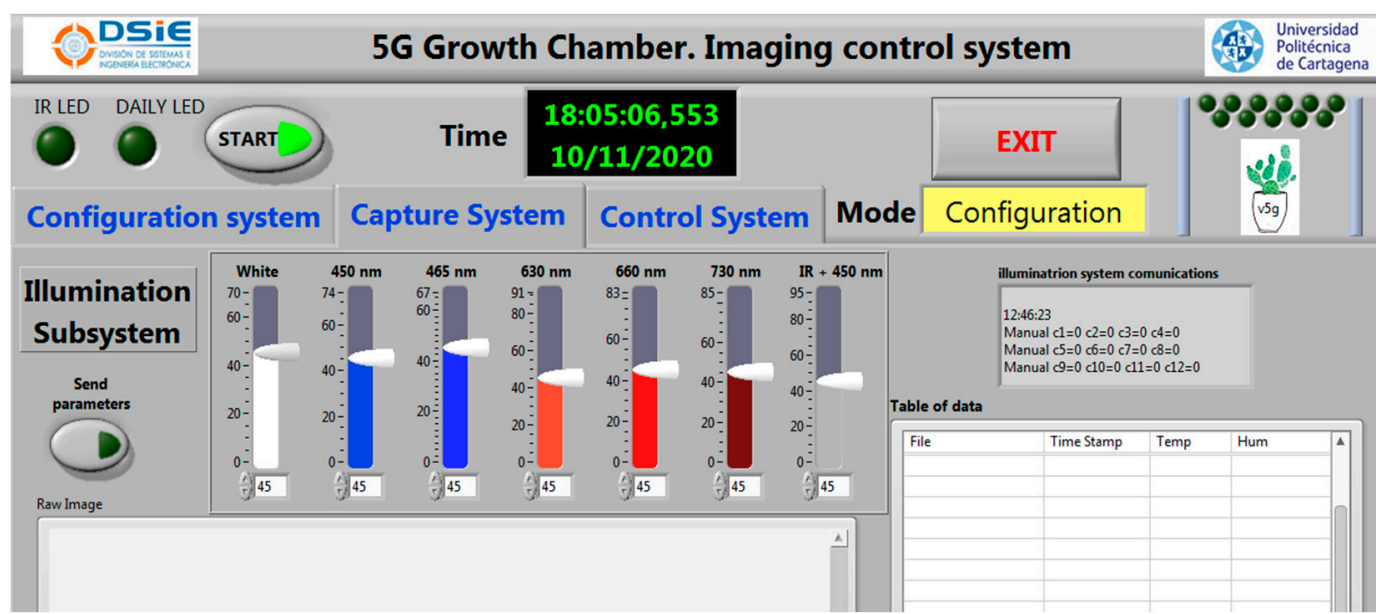

Figure 4. Software interface for configuration and control of the multispectral computer vision system. 


\subsection{MSI Acquisition Process and Image Pre-Processing}

\subsubsection{MSI Acquisition Process}

In the multispectral image acquisition process, 1810 berries of five varieties of seedless table grape have been used: 408 grains of Autumn Royal, 602 grains of Crimson Seedless, 196 grains of Itum4, 408 grains Itum 5 and 196 grains of Itum9. Figure 5 shows an example of the appearance of a bunch of each variety and an example of the samples used in the vision system. The five varieties used show three colours. Autumn Royal is dark red, Crimson Seedless and Itum 9 are red and Itum 4 and Itum 5 are green. To the human eye, Autumn Royal is clearly darker than the rest. However, it is very difficult to discriminate between Crimson Seedless and Itum 9 and between Itum 4 and Itum5.

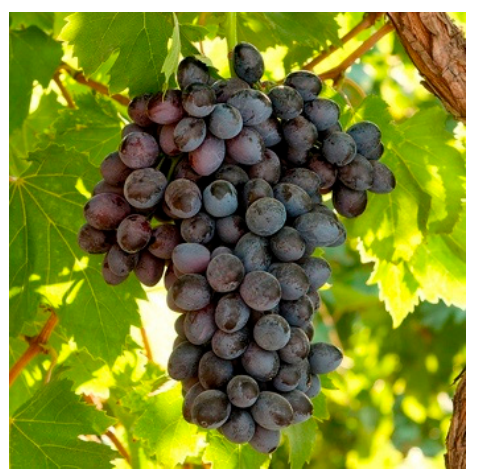

(a)

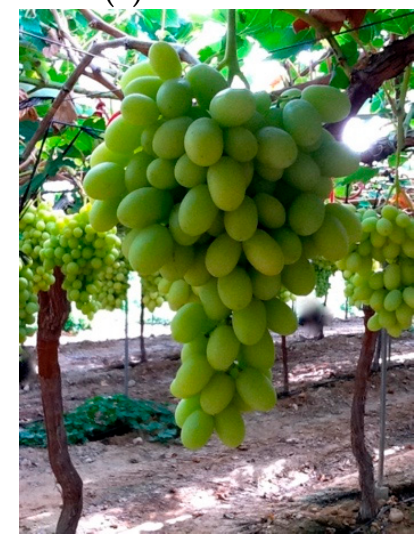

(d)

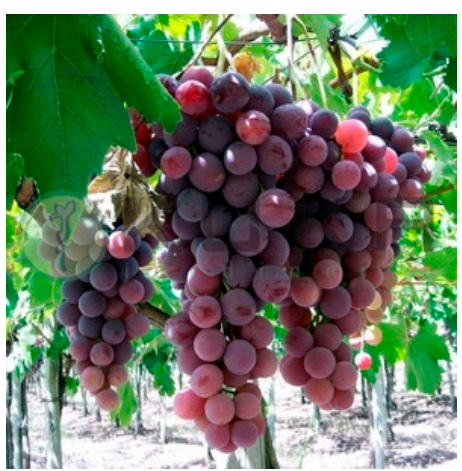

(b)

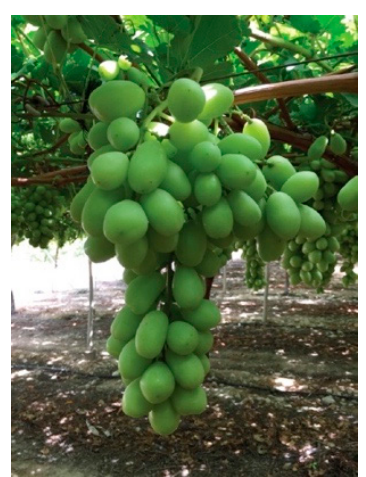

(c)

Figure 5. Varieties of grape grains used in the MIS acquisition process: (a) Autumn Royal, (b) Crimson Seedless, (c) Itum 4, (d) Itum 5 and (e) Itum 9.

Each grape grain was photographed with the VIS and IR cameras shown in Section 2.1.2. The VIS camera was configured to capture 12 bands that correspond to the spectral wavelengths in nanometres: $488.38,488.58,503.59,516.60,530.62,542.17,567.96,579.29,592.89$, $602.88,616.59$ and 625.71 . The IR camera was configured to capture 25 bands that correspond to the spectral wavelength in nanometres: $676.25,689.83,714.87,729.06,741.80$, $755.44,767.48,781.25,792.57,805.25,823.75,835.10,845.81,856.52,866.88,876.49,885.18$, $894.26,908.58,916.63,925.36,931.99,938.48,946.14$ and 952.76 .

Figure $6 \mathrm{a}, \mathrm{b}$ show the capturing process for the VIS and IR cameras used in the dark chamber. A white reference marker of known dimensions is used in each image for future exact measurements of shape and dimensional calibration. 


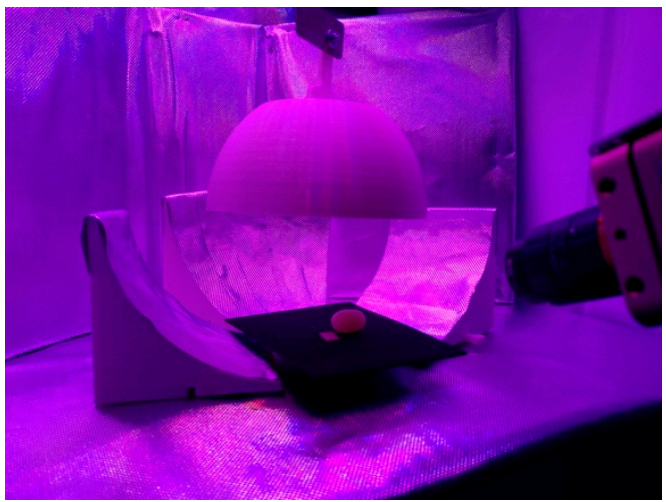

(a)

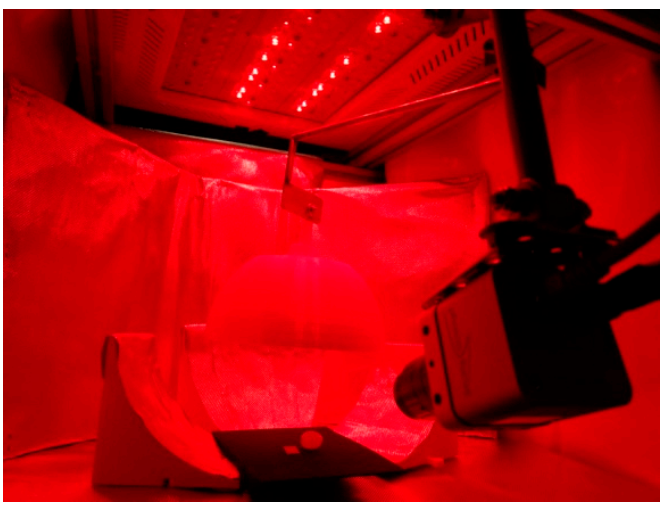

(b)

Figure 6. Configuration of the dark chamber for capturing grape images: (a) visible and (b) IR.

Figure 7a,b show the 12 VIS images and 25 IR images, respectively, calibrated using Equation (1) for the grape variety Itum5.

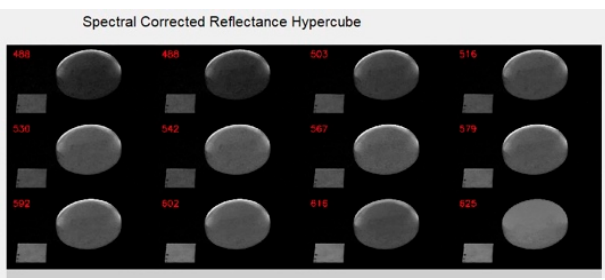

(a)

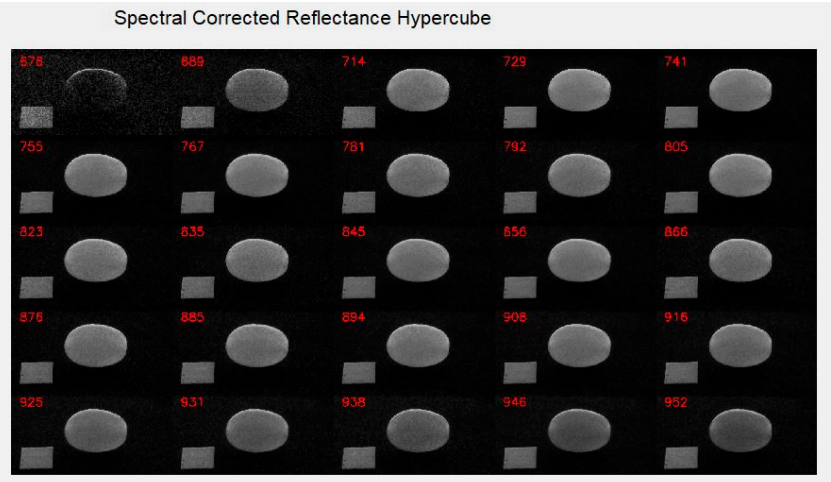

(b)

Figure 7. (a) 12 bands of grape variety Itum 5 obtained in the visible spectrum and (b) 25 bands of grape variety Itum5 obtained in the IR spectrum.

\subsubsection{Image Pre-Processing}

The images captured in the image acquisition process have been pre-processed before being supplied to the Deep Neural Network (DNN) algorithms. The aim of this stage is to separate the image of the grape grain from the background to obtain the most precise spectral information possible, without noise or elements in the image other than the grape. To achieve this, an automatic segmentation algorithm was developed that searches all of the bands in an MSI for the object that most closely resembles a grape using shape characteristics. Then the region that contains the grape is used to extract the grapes from all the bands. The algorithm developed is made up of a processing pipeline of basic computer vision methods, which are described below:

1. Load an MSI consisting of $\mathrm{N}$ bands: $\mathrm{MSI}=\left\{B_{0}, \ldots, B_{N}\right\}$.

2. Calculate the mean Shannon entropy for all the bands of the MSI (MSE), as well as for each band: $\left\{\mathrm{SE}_{0}, \ldots, \mathrm{SE}_{\mathrm{N}}\right\}$.

3. For each entropy value $\mathrm{SE}_{\mathrm{i}}$ :

a. If $\mathrm{MSE}>\mathrm{SE}_{\mathrm{i}}$, we consider that the image has an acceptable information distribution and the Otsu [27] thresholding method will be applied, obtaining a new set of black-and-white images $\left\{\mathrm{BW}_{0}, \ldots, \mathrm{BW}_{\mathrm{N}}\right\}$. 
b. Otherwise, we consider that the image has a low information distribution, and the bands will be limited with a threshold value of 10 , obtaining a new set of black-and-white images $\left\{\mathrm{BW}_{0}, \ldots, \mathrm{BW}_{\mathrm{N}}\right\}$.

c. A contour search algorithm will be applied to the images $\left\{\mathrm{BW}_{0}, \ldots, \mathrm{BW}_{\mathrm{N}}\right\}$, obtaining a new set of contours $\left\{C_{0}, \ldots, C_{M}\right\}$. From the set, those contours $\left\{C_{0}, \ldots, C_{K}\right\}$ that verify the area and roundness restriction criteria given by Equation (2) will be selected:

$$
C_{j} \in\left\{C_{0}, \ldots C_{K}\right\} \text { if }\left\{\begin{array}{c}
\left.8000<\operatorname{area}\left(C_{j}\right)<40000\right) \\
\bigwedge_{\left.0.4<\text { circularity }\left(C_{j}\right)<1.2\right)}
\end{array}\right.
$$

4. From the set of contours $\left\{C_{0}, \ldots, C_{j}, \ldots, C_{M}\right\}$, which verify the area and roundness restrictions, the one with the largest area, $C_{m}$, will be selected as the best segmentation of the grape. The window containing the contour $C_{m}(y: y+h, x: x+w)$ will be used to segment all of the grapes of each band of the MSI, where $(y, x)$ is the upper-left corner of the rectangle, $h$ the height and $w$ the width.

5. Each MSI has been resized to a size of $140 \times 200$ pixels.

6. Go to step 1.

Figure $8 \mathrm{a}, \mathrm{b}$ show the result of the pre-processing applied to a visible MSI and an MSI captured in IR, respectively.

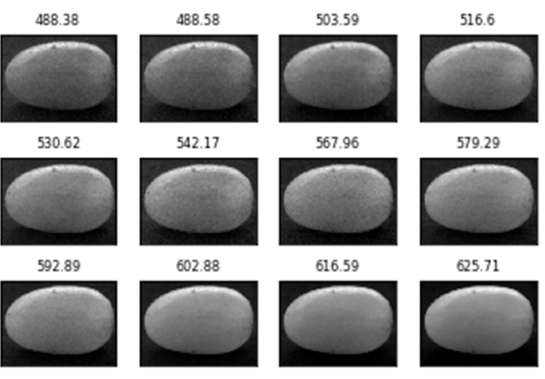

(a)

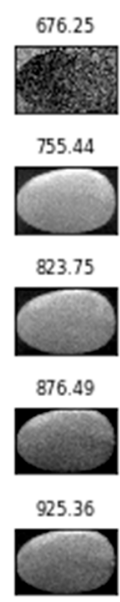

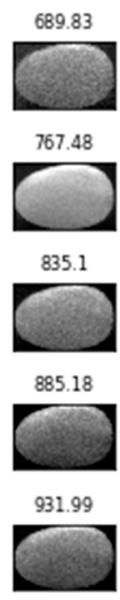

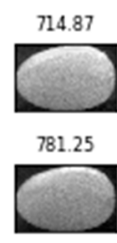

845.81

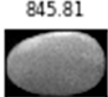

894.26

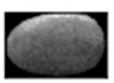

938.48

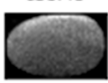

(b)
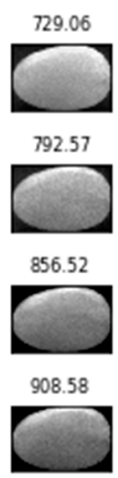

946.14
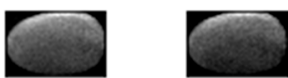

Figure 8. (a) 12 pre-processed bands obtained in the visible spectrum and (b) 25 pre-processed bands obtained in the IR spectrum. The numbers above the images correspond to the wavelength of image acquisition.

\subsubsection{Data Augmentation}

The original dataset was split into training and validation subsets of 1358 and 452 images, that account for $75 \%$ and $25 \%$ of the images, respectively. The class distribution in both subsets is the same, as the test subset contains $25 \%$ of the images of each of the five different grape varieties used.

To increase the number of training images, a data augmentation pipeline was developed using the Python library API, Albumentations [28]. The new augmented images were generated by sequentially applying the following transformations with a set probability of $50 \%$ each: either horizontal or vertical flip, random contrast and brightness alteration, and affine transformation. This last one includes horizontal and vertical shifts, rotation and scaling of the images. All the transformations were controlled with parameter values selected randomly within a defined range, except for the horizontal and vertical flip (Table 2). 
Table 2. Transformation ranges of data augmentation.

\begin{tabular}{cc}
\hline Transformation & Range \\
\hline Brightness & $(-0.15,+0.15)$ \\
Contrast & $(-0.15,+0.15)$ \\
Rotation & $\left(-90,+90^{\circ}\right)$ \\
$X$ axis shift & $(-0.006,+0.006)$ \\
$Y$ axis shift & $(-0.006,+0.006)$ \\
Scale & $(-0.3,+0.3)$ \\
\hline
\end{tabular}

The data augmentation pipeline was implemented in a generator-type Python function, and therefore, the new data were not stored but generated in fixed-size batches in each training step of the classification models (see Figure 9). The function randomly selects a number of images from the training subset equal to the batch size and then applies the augmentation pipeline described earlier to every image. After a batch of augmented images is created and before it is delivered to the training algorithm, it is normalised to a range of pixel values from 0 to 1, by dividing by 255 the maximum possible value in 1-byte resolution images.

The test and training datasets were augmented with the same augmentation pipeline. As the data are already separated into two subsets before being fed to the generators, there is no risk that the test generator will produce images equal or very similar to the ones that have been generated by the training generator, which could lead to lower loss values in the test dataset and erroneous conclusions about the performance of the models. The class distributions of both augmented datasets are the same because of the random selection of images to transform from the dataset and, as mentioned earlier, the subsets have been created respecting the class distribution.

The total number of images generated in any training epoch is equal to the batch size, 16 times the number of steps per epoch, which were 500 for the training phase and 150 for the validation, making a total of 8000 images for training and 2400 for validating. Finally, after fitting, each model was tested using the 1810 original images without any augmentation.

The following diagram illustrates the whole process.

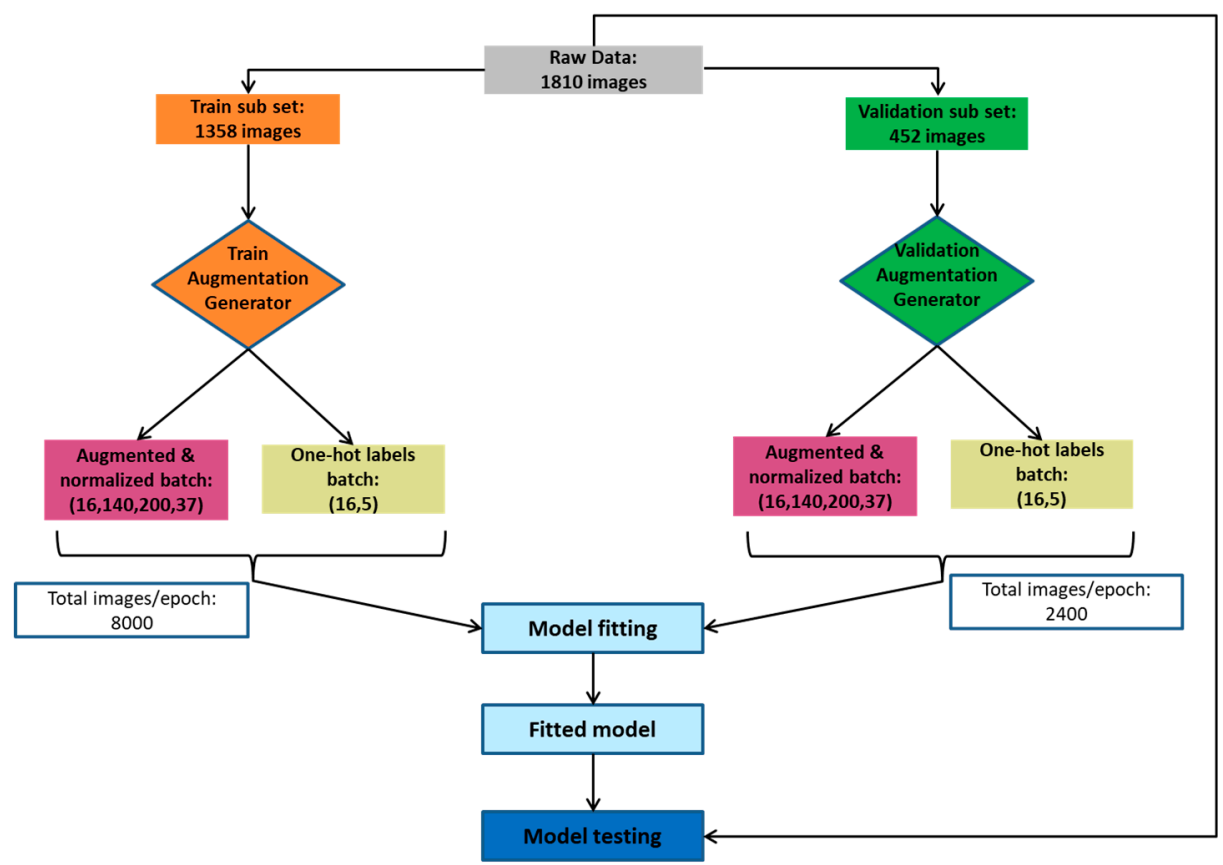

Figure 9. Workflow of the data augmentation process. 


\subsection{Deep Learning Methods}

Methods based on deep learning belong to the group of algorithms associated with machine learning and have proven their effectiveness in various fields of science; in addition there is a growing interest from the scientific and research communities. Among the most used methods are (1) convolutional neural networks (CNNs), (2) recurrent neural networks (RNNs), (3) generative adversarial networks, (4) Boltzmann machines, (5) deep reinforcement and (6) autoencoders.

In this work, we used CNNs as the tool to perform the multispectral image classification of five varieties of grape grains. CNNs were designed to avoid the tedious feature extraction processes that computer vision experts previously used to carry out the segmentation and classification of objects in images. CNNs have the capacity to automatically extract the most relevant characteristics that minimise a certain function, $f^{*}$. The goal of the $\mathrm{CNN}$ classification model $y=f^{*}(x)$ is to map an input $x$ to a category $y$. The network is capable of learning a set of parameters, $\theta$, that obtain the best function approximation $y=f^{*}(x ; \theta)[29]$.

At the architecture level, CNNs are composed of different layers (L), which are grouped into blocks that can be distributed into different branches and unions. Figure 10 shows some of the most common blocks used to create $\mathrm{CNN}$ architectures. The advantages of these architectures are that once trained, they can be reused for different applications.

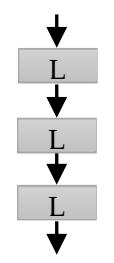

(a)

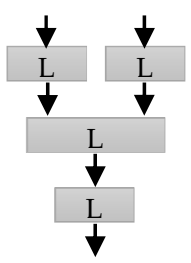

(b)

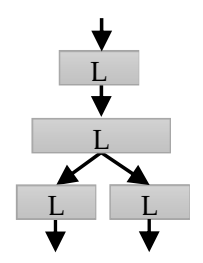

(c)

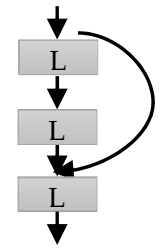

(d)

Figure 10. (a) Sequential block, (b) concatenation block (c) expansion block and (d) residual inception block.

To solve a certain classification problem, the blocks shown in Figure 10 are selected and assembled with the aim of forming an architecture that can optimise the mapping of input images in one or more output classes. The most used layers in these blocks that form the $\mathrm{CNN}$ architectures are described below:

- Input layer. This is the data input layer of the $\mathrm{CNN}$ and is composed of the normalised training images. These images can have a single channel or multiple channels, such as multispectral, video or medical images (i.e., magnetic resonance imaging (MRI) and computerised tomography (CT) scans).

- Convolutional layers. These units have the ability to extract the most relevant characteristics from the images. The convolutions on the images are usually 2D. Given an image I and a convolution kernel $\mathrm{K}$, the 2D convolution operation is defined by Equation (3).

$$
\mathrm{S}(\mathrm{i}, \mathbf{j})=(\mathrm{I} * \mathrm{~K})(\mathbf{i}, \mathbf{j})=\sum_{m} \sum_{n} I(m, n) K(i-m, j-n)
$$

Equation (3) allows the extraction of spatial features from images in 2D. However, there are other convolutions that exploit the multidimensional relations present in images with either 2 or 3 dimensions. These are called 3D convolutions [30], and the main difference compared to the $2 \mathrm{D}$ convolutions is that the kernel used has 3 dimensions instead of 2. This can be imagined as a data cube $(k \times k \times d)$, which would be the filter that moves along the 3 spatial axes of an image with a given stride and performs the dot product between the pixel values of the image and the numbers in the filter, at each step, as can be seen in Figure 11. 


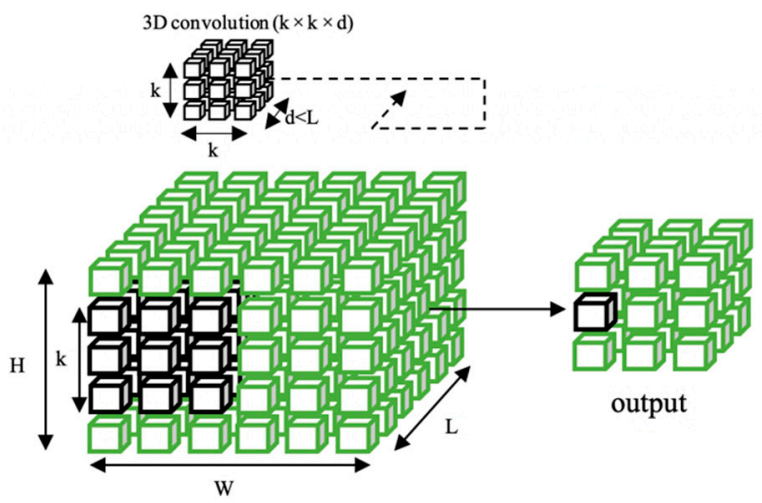

Figure 11. Diagram of a 3D convolution operation [30].

To apply a 3D convolution to a 2D image of size $W \times H \times L$ (width pixels times height pixels times the number of channels), the array that constitutes the image must be reshaped so that the channel axis is interpreted as a third depth axis, and a fourth axis of size 1 is added, which would be the channel axis of a proper 3D image that contains the pixel values. In the case of a normal 2D image defined with the common RGB colour map, there is likely little to gain by applying 3D convolutions, because the channel axis is only of depth 3, and as such, there is not much information stored in it compared to the spatial axes of larger sizes. Thus, the channel axis is larger and contains more information about the object being captured than the channel axis of an RGB image, namely the reflectance at various wavelengths. Therefore, in this case, applying 3D convolutions can lead to better results with a deep learning model using fewer convolutional layers and, in turn, fewer parameters.

- $\quad$ Fully connected layers. These layers are those layers where all the inputs from one layer are connected to every unit of the next layer. They have the capacity to make decisions, and in our architecture, they will carry out the classification of the data into various classes.

- Activation layer. It has the capacity to apply a nonlinear function as output of the neurons [31]. The most commonly used activation function is the rectified linear unit $(\operatorname{ReLU})$, and it is defined in Equation (4).

$$
\operatorname{ReLU}(x)= \begin{cases}x, & x \geq 0 \\ 0, & x<0\end{cases}
$$

- Batch normalisation layer. This layer standardises the mean and variance of each unit in order to stabilise learning, reducing the number of training epochs required to train deep networks [29].

- Pooling layer. The pooling layer modifies the output of the network at a certain location with a summary statistic of the nearby outputs, and it produces a down-sampling operation over the network parameters. For example, the Max Pooling layer uses the maximum value from each of the clusters of neurons at the prior layer [32]. Another common pooling operation is the calculation of the mean value of each possible cluster of values of the tensor of the previous layer, which is implemented in the Average Pooling layer. Both of these kinds of pooling operations can be applied to the whole tensor of the prior layer instead of just a cluster of its values in a sliding window that moves along it. These layers are called Global Max Pooling and Global Average Pooling, and they reduce a tensor to its maximum or mean value, respectively. Additionally, every pooling operation can be applied two- or three-dimensionally, which means that they act along the first 2 or 3 axes of the given tensor. For instance, a 3D Global Average Pooling layer would reduce a tensor of size $w \times h \times d \times f$ (width times height times depth times features) to a $1 \times f$ one, thus spatially compressing the information of every feature to just one value, its mean. 
- Output layer. In CNNs for classification, this layer is formed by the last layer of the fully connected block and contains the activation layer, which obtains the probability of belonging to a particular class.

\subsubsection{Pretrained DL Architectures}

Over the course of the last decades, there have been many advances in the design of CNN architectures, which have been increasing in depth and complexity, allowing for impressive results in many deep learning projects. Some of the most well-known architectures include LeNet-5, AlexNet, VGG16, ResNet and the Inception variants, which will be briefly reviewed below.

- LeNet-5 was published in 1998 and was originally conceived to recognise handwritten digits in banking documents. It is one of the first widely used CNN and has served as the foundation for many of the more recently developed architectures. The original design consisted of two 2D convolution layers with a kernel size of $5 \times 5$, each one followed by an average pooling layer, after which a flattening layer and 3 dense layers were placed, the last one being the output layer. It did not make use of batch normalisation and the activation layer was tan $h$ instead of the now widely used ReLU [33].

- AlexNet was published in 2012 and won the contest of ImageNet LSVRC-2010, which consisted of the classification of 1.2 million images of 1000 distinct classes. The network has a significantly larger number of parameters compared to LeNet-5, about 60 million. It consists of a total of five 2D convolutional layers, of varying kernel size that decrease with the depth of the layer: $11 \times 11,5 \times 5$ and $3 \times 3$ for the last 3 . In between the first three convolutional layers and after the last one, there are a total of 4 Max Pooling layers. Then, a flattening layer and three dense layers follow, including the final output layer. The innovations of this CNN are the usage of the ReLU activation function and the dropout layers to reduce overfitting. Also, the usage of GPUs to train any CNN model with more than a million parameters, which is now commonplace, can be traced back to the inception of this architecture [34].

- VGG16 was published in 2014 as a result of the investigation of the effect of the kernel size on the results achieved with a deep learning model. It was found that with small kernel sizes of $3 \times 3$ but an increased number of convolutional layers, 16 in this case, the performance experienced a significant improvement. This architecture won the ImageNet challenge of 2014 and consists of 16 convolutional layers, all with kernel size $3 \times 3$, grouped in blocks of two or three layers each. The groups are followed by Max Pooling layers, and after the last convolution block, there are three dense layers, including the output layer. This architecture showed that the deeper the CNN is, the better the results achieved by it usually are, but the memory requirements also increase and must be taken into account. The number of parameters of this architecture is very high, 137 million, but the authors created an even deeper network, called VGG19, with 144 million parameters [35].

- Inception was published in 2014 and competed with VGG16 in the same ImageNet challenge, where it achieved results as impressive as those of VGG16 but using far less parameters, only 5 million. To accomplish this, the architecture makes use of the so-called inception blocks, which consist of 4 convolutions applied in a parallel manner to the input of the block, each one of a different kernel size, and whose outputs are, in turn, concatenated. The network is made of 3 stacked convolutional layers and then 9 Inception blocks, followed by the output layer. There are also 2 so-called auxiliary classifiers that emerge from 2 Inception blocks, whose purpose is to mitigate the vanishing gradient problem that accompanies a large neuronal network like this. These two branches are discarded at inference time, being used only during training. The kernel sizes of all the convolutional layers were chosen by the authors to optimise the computational resources. After this architecture was published, the authors designed improved versions like InceptionV3 in 2015 and Inception V4 in 
2016. InceptionV3 focused on improving the computation efficiency and elimination of representational bottlenecks [36], and InceptionV4 took some inspiration from the ResNet architecture and combined its residual connections, which will be briefly discussed below, with the core ideas of InceptionV3 [37]. In 2016, yet another variant of Inception was published, called Xception. Its main feature is the replacement of all the convolutions by depth-wise separable convolutions to increase the efficiency even further [38].

- ResNet was published in 2015 and won the first place of the ILSVRC classification task of the year. The main new feature of this architecture are the skip connections and the consistent usage of batch normalisation layers after every convolutional layer. The purpose of these features is to ease the training of very deep CNNs, like the implementations ResNet-50 and ResNet-101, which have 50 and 101 convolutional layers, respectively. The architecture is composed of so-called convolutional and identity blocks, in which the input to the block is concatenated to its output, creating the skip connections that help to train the networks [39].

All of these architectures have been used in the field of remote sensing in numerous studies. In the case of LeNet-5, for example, in [40], the authors used a slightly modified version of this $\mathrm{CNN}$, among others, to track the eyes of typhoons with satellite IR imaging; and in [41], they created, as part of their study, a pretrained LeNet-5 model using the UC Merced Dataset to classify cropland images. AlexNet is used in [42] to classify the images of the UCM spaceflight, dataset and in [43], a pretrained AlexNet model is fine-tuned to classify wetland images. VGG16 and 19, InceptionV3, Xception and ResNet50, among others, are used in a comparative study [44] to classify complex multispectral optical imagery of wetlands. In [45], the authors designed an improved version of InceptionV3 to classify ship imagery from optical remote sensors. This architecture is also the base of the one used in [46] to classify images of damaged buildings by earthquakes using highresolution remote sensing images; and in [47], the authors pretrained an ImageNet2015 InceptionV3 model together with VGG19 and ResNet50 to classify images of high spatial resolution. The Xception architecture was used in [48] to detect palm oil plantations, and ResNet50 was used in [49] to detect airports in large remote sensing images. These are just a few examples of the many existing studies in which $\mathrm{CNN}$ algorithms are used.

\subsubsection{Ad hoc Deep Learning Architecture Design}

To perform the classification of the five grape varieties, two custom architectures have been designed, consisting of the following layers: (1) a normalised input layer; (2) two 3D filter blocks made up of 3D convolutional layers (3D:d,k,k,k), a ReLU activation function, Bath normalisation and 3D Pooling layers (Filter\#1: 3DAveragePooling (3D:AP); Filter\#2: 3D GlobalAveragePolling (3D:GAP)); (3) a fully connected (FC) layer; and (4) an output (O) layer. The first architecture (Figure 12a) is formed by a sequential structure of layers with a single-branch output (SBO), while the second proposed architecture is similar to the first (Figure 12b) but has an output distributed as a multiple-branch output (MBO). Both architectures have been designed to exploit the multidimensionality present in multispectral images, for which a 3D filter block has been designed consisting of four layers (see Figure 12a,b), which has allowed the extraction of spatial-spectral features of the different bands captured by the vision system to be maximised.

With the aim of obtaining an optimal design for the parameters of the $\mathrm{SBO}$ and $\mathrm{MBO}$ architectures regarding the 3D filter banks, two types of test have been designed:

(1) Optimal 3D kernel size. In this test, both architectures were evaluated with symmetric kernel sizes: $(5 \times 5 \times 5),(7 \times 7 \times 7)$ and $(10 \times 10 \times 10)$. The different kernels will allow different space-time relationships to be captured for the multispectral images and the evaluation of the size that produces the best result in the classification process.

(2) Optimal kernel sequence: In this experiment, architectures with three types of kernel sequences were evaluated: increasing F\#1 $(5 \times 5 \times 5)+\mathrm{F} \# 2(10 \times 10 \times 10)$, decreasing $\mathrm{F} \# 1(10 \times 10 \times 10)+\mathrm{F} \# 2(5 \times 5 \times 5)$ and constant F\#1 $(7 \times 7 \times 7)+\mathrm{F} \# 2(7 \times 7 \times 7)$. The 
results will allow the influence of the sequence order of the kernels on the classification process to be determined.

Both tests have been performed with a kernel depth of $d=16$ and have used a dilation rate of $2 \times 2 \times 2$ in the $3 \mathrm{D}$ convolutional layer.

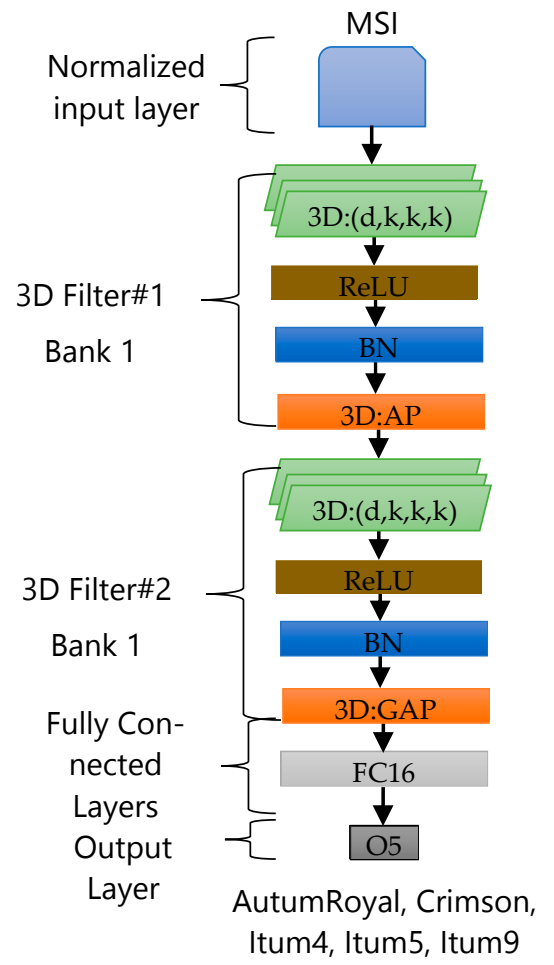

(a)

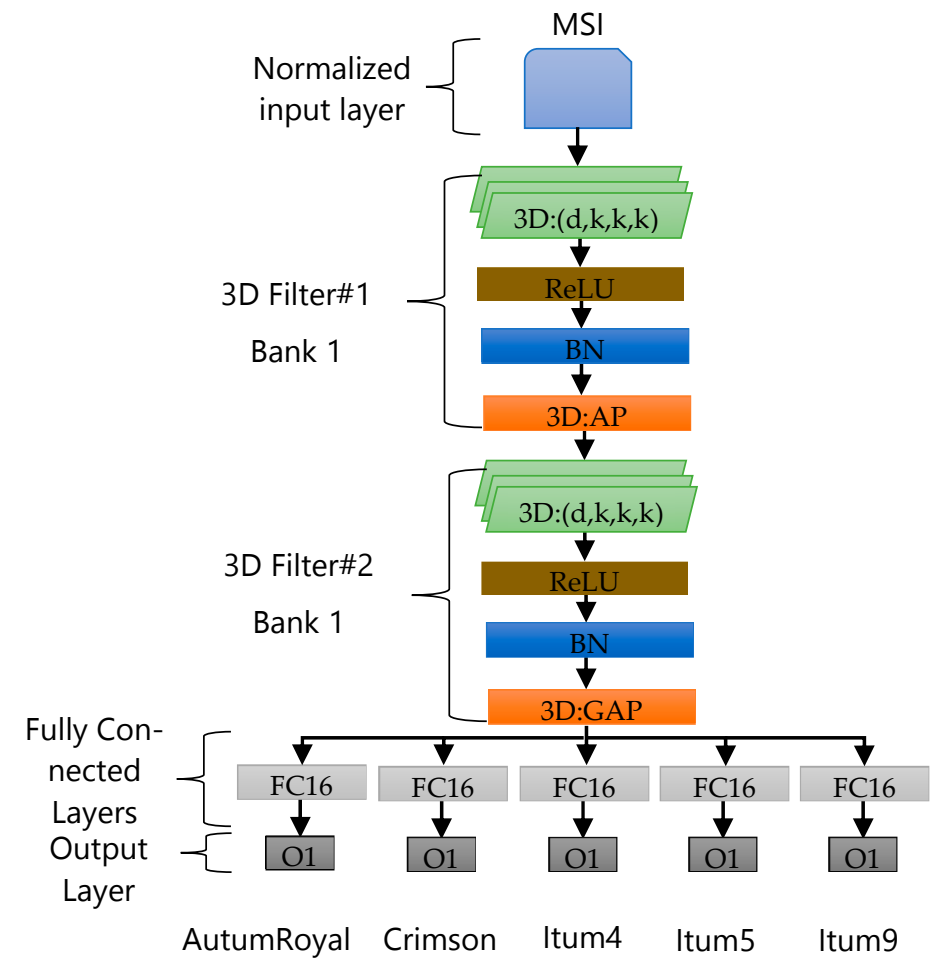

(b)

Figure 12. Proposed architectures with (a) single-branch output (SBO) and (b) multiple-branch output (MBO).

Table 3 shows the total number parameters for each architecture and per layer that have been used after configuring the architectures in Figure 12 according to the tests designed for calculating the optimal number of parameters.

Table 3. Number of parameters per layer and total of SBO and MBO architectures.

\begin{tabular}{|c|c|c|c|c|c|c|c|}
\hline & $\begin{array}{c}\text { Architecture } \\
\text { Layer }\end{array}$ & $\begin{array}{c}\text { SBO } \\
(5 \times 5 \times 5) \\
{[(0 \times 10 \times 10)} \\
\end{array}$ & $\begin{array}{c}\text { SBO } \\
(10 \times 10 \times 10) \\
(5 \times 5 \times 5)\end{array}$ & $\begin{array}{c}\text { SBO } \\
(7 \times 7 \times 7) \\
(7 \times 7 \times 7) \\
\end{array}$ & $\begin{array}{c}\text { MBO } \\
(5 \times 5 \times 5) \\
(10 \times 10 \times 10) \\
\end{array}$ & $\begin{array}{c}\text { MBO } \\
(10 \times 10 \times 10) \\
(5 \times 5 \times 5)\end{array}$ & $\begin{array}{c}\text { MBO } \\
(7 \times 7 \times 7) \\
(7 \times 7 \times 7) \\
\end{array}$ \\
\hline \multirow{5}{*}{ 暮 } & Input & 0 & 0 & 0 & 0 & 0 & 0 \\
\hline & 3D:Conv & 1008 & 8008 & 2752 & 1008 & 8008 & 2752 \\
\hline & ReLU & 0 & 0 & 0 & 0 & 0 & 0 \\
\hline & BN & 32 & 32 & 32 & 32 & 32 & 32 \\
\hline & 3D:MP & 0 & 0 & 0 & 0 & 0 & 0 \\
\hline \multirow{6}{*}{ 䓪 } & 3D:Conv & 128,016 & 16,016 & 43,920 & 128,016 & 16,016 & 43,920 \\
\hline & ReLU & 0 & 0 & 0 & 0 & 0 & 0 \\
\hline & BN & 64 & 64 & 64 & 64 & 64 & 64 \\
\hline & 3D:GAP & 0 & 0 & 0 & 0 & 0 & 0 \\
\hline & FC16 & 272 & 272 & 272 & $1360^{1}$ & $1360^{1}$ & $1360^{1}$ \\
\hline & Output & 85 & 85 & 85 & $85^{2}$ & $85^{2}$ & $85^{2}$ \\
\hline \multicolumn{2}{|c|}{ Total Parameters } & 129,477 & 24,477 & 47,125 & 130,565 & 25,565 & 48,213 \\
\hline
\end{tabular}

\footnotetext{
${ }^{1}$ Five FC16 branches: $272 \times 5=1360 .{ }^{2}$ Five outputs: $17 \times 5=85$.
} 


\subsection{Plant Material}

All grape varieties used in the current study were grown in a commercial orchard (Cooperativa Las Cabezuelas), where seedless grapes and Narcissus are grown for export under ecological conditions [50]. Seedless grapes are of the commercial varieties Crimson Seedless, Autumn Royal, Itum4, Itum5 and Itum9. While Crimson Seedless and Autumn Royal are classic seedless grapes, the Itum series correspond to a new selection created recently for high-quality table grapes [51]. All seedless grapes share a common mutation rendering stenospermocarpic seed abortion [52]. Grapes were harvested when ready to market. Starting in mid-June and till late December, different varieties were transported to the lab and conserved in a cold chamber at $6^{\circ}$ during the image acquisition period.

\section{Results}

In the design and implementation phase of the deep learning architectures for the classification of multispectral images, the Keras 2.3.1 under TensorFlow 2.0.0 libraries have been used. The Albumentation library [28] has been used in the data augmentation process for multichannel images, and the OpenCV library [53] has been used in the implementation of the image pre-processing algorithm (Section 2.2.2). The methods proposed were programmed in Python 3.0 with the Spyder 3.0 IDE running on a computer installed with Windows 10 Professional with Intel Core ${ }^{\mathrm{TM}}$ processor i7-7700K at $4.20 \mathrm{GHz}, 64 \mathrm{~GB}$ DDR4 and dedicated graphics NVIDIA GeForce 1080 with 8 GB memory.

\subsection{Configuration of the Models}

Three multispectral datasets were used for obtaining the classification models of the five grape varieties with each of the configurations proposed for each architecture.

(1) Training dataset, which consists of 8000 images, $75 \%$ of the images obtained in the data augmentation process (Section 2.2.2)

(2) Validation dataset, consisting of 2400 images, which corresponds to $25 \%$ of the images obtained in the data augmentation process (Section 2.2.2)

(3) Test dataset, made up of 1810 images, which constitute the set of images without the initial data augmentation

Table 4 shows the hyperparameters used to configure the training and validation phases of all the classification models developed in this work.

Table 4. Hyperparameter configuration.

\begin{tabular}{lc}
\hline Parameter Name & Value \\
\hline Batch size & 16 \\
Number of epochs & 20 \\
Optimisation algorithm & Adam \\
Loss function & Categorical cross Entropy \\
Metric & Accuracy \\
Learning rate & 0.001 \\
Momentum & None \\
Activation function in convolutional layers & ReLU \\
Activation function in last layers & Softmax \\
\hline
\end{tabular}

\subsection{Performance Metrics}

To evaluate the performance of the proposed architectures, the metrics accuracy in percentage and the loss function error were calculated during the training and validation of the models. The accuracy in percentage was used to evaluate the test dataset. In addition, the confusion matrix was calculated to obtain more precise information about the classification process of the grape varieties.

Table 5 shows the accuracy and loss values of the different tests performed with the datasets (training, validation and test) used for training, validation and testing of the 
models for each of the settings in the filter banks $((5 \times 5 \times 5)+(10 \times 10 \times 10),(10 \times 10$ $\times 10)+(5 \times 5 \times 5)$ and $(7 \times 7 \times 7)+(7 \times 7 \times 7))$ established in the SBO and MBO architectures.

Table 5. Training, validation and test accuracy and loss function error for three different filter sequences of SBO and MBO architectures.

\begin{tabular}{|c|c|c|c|c|c|c|}
\hline \multirow{2}{*}{$\begin{array}{c}\text { Architecture } \\
\text { Metrics }\end{array}$} & \multicolumn{3}{|c|}{ SBO Kernel Sizes } & \multicolumn{3}{|c|}{ MBO Kernel Sizes } \\
\hline & $\begin{array}{c}(5 \times 5 \times 5) \\
(10 \times 10 \times 10)\end{array}$ & $\begin{array}{c}(10 \times 10 \times 10) \\
(5 \times 5 \times 5)\end{array}$ & $\begin{array}{l}(7 \times 7 \times 7) \\
(7 \times 7 \times 7)\end{array}$ & $\begin{array}{c}(5 \times 5 \times 5) \\
(10 \times 10 \times 10)\end{array}$ & $\begin{array}{c}(10 \times 10 \times 10) \\
(5 \times 5 \times 5)\end{array}$ & $\begin{array}{l}(7 \times 7 \times 7) \\
(7 \times 7 \times 7)\end{array}$ \\
\hline Train. acc. $\{\%)$ & 100.00 & 100.00 & 100.00 & 100.00 & 99.89 & 100.00 \\
\hline Train. loss & 0.00072 & 0.0063 & 0.00030 & 0.00035 & 0.01379 & 0.00069 \\
\hline Val. acc. (\%) & 100.00 & 82.708 & 100.00 & 100.00 & 100.00 & 100.00 \\
\hline Epoch & 11 & 14 & 7 & 10 & 13 & 15 \\
\hline Val. loss & 0.00202 & 0.583 & $3.693 \times 10^{-5}$ & 0.00012 & 0.00018 & 0.00025 \\
\hline Test acc. (\%) & 100.00 & 83.20 & 100.00 & 100.00 & 100.0 & 100.00 \\
\hline
\end{tabular}

The results shown in Table 5 confirm that the designs proposed for the SBO and MBO architectures based on two 3D filter blocks managed to classify $100 \%$ of the grape berries correctly. With the validation and testing sets, a rating of $100 \%$ was obtained with all the proposed architectures except with the SBO architecture and kernel sizes: F\#1(10 × 10 $\times 10)+\mathrm{F} \# 2(5 \times 5 \times 5)$.

As for the relationship between the distribution of kernel sizes in 3D filter blocks and the number of epochs, it should be noted that the constant kernel sequence $\mathrm{F} \# 1(7 \times 7 \times 7)$ $+\mathrm{F} \# 2(7 \times 7 \times 7)$ achieved a maximum rating value with only 7 epochs out of a total of 20 .

In addition, in Figure 1, it can be observed that the data augmentation process followed in Section 2.2.2 by which a set of 10,400 multispectral images have been generated, starting from a set of 1810 images captured in the dark chamber, allowed models that have been able to generalise and classify all of the original images in the test set to be obtained.

Tables 6 and 7 show the confusion matrices for the test dataset. The results in both tables show that the SBO and MBO architectures do not show any difference when classifying $100 \%$ of the five grape varieties with increasing and constant kernel sequences. On the other hand, the decreasing kernel sequence $((10 \times 10 \times 10)+(5 \times 5 \times 5))$ obtained worse results in the classification for both architectures. The MBO architecture offers a $99.40 \%$ correct classification compared to $83.20 \%$ for the SBO architecture; specifically, the SBO architecture had the biggest problems classifying the Crimson Seedless, Itum5 and Itum 9 varieties.

Finally, and from the values in Table 3, the total number of parameters for each of the configurations of the SBO $(129,477,24,477,47,125)$ and MBO $(130,565,25,565,48,213)$ architectures do not show significant differences. The architecture with the most parameters is $\mathrm{MBO}$ with an increasing kernel sequence F\#1 $(5 \times 5 \times 5)+\mathrm{F} \# 2(10 \times 10 \times 10)$, and that with the least parameters is the SBO with a decreasing kernel sequence F\#1 $(10 \times 10 \times 10)+$ F\#2 $(5 \times 5 \times 5)$.

From the results obtained in this section, the optimal architecture according to classification performance $(100 \%)$ and number of parameters $(25,565)$ is the MBO with decreasing kernel sizes F\#1 $(10 \times 10 \times 10)+\mathrm{F} \# 2(5 \times 5 \times 5)$.

From here on, the architecture optimised for the classification of multispectral images based on 3D filter banks will be referred to as 3DeepM. 
Table 6. Confusion matrix for the three configurations of the 3D convolution layer in the SBO architecture over test data.

\begin{tabular}{|c|c|c|c|c|c|c|}
\hline 3D Conv. & Variety & Autumn Royal & Crimson Seedless & Itum 4 & Itum5 & Itum9 \\
\hline \multirow{5}{*}{$\begin{array}{c}\text { F\#1(5 × } 5 \times 5) \\
\text { F\#2(10 × } 10 \times 10)\end{array}$} & Autumn Royal & $100 \%$ & 0 & 0 & 0 & 0 \\
\hline & Crimson & 0 & $100 \%$ & 0 & 0 & 0 \\
\hline & Itum4 & 0 & 0 & $100 \%$ & 0 & 0 \\
\hline & Itum5 & 0 & 0 & 0 & $100 \%$ & 0 \\
\hline & Itum9 & 0 & 0 & 0 & 0 & $100 \%$ \\
\hline \multirow{5}{*}{$\begin{array}{c}\mathrm{F} \# 1(10 \times 10 \times 10) \\
\mathrm{F} \# 2(5 \times 5 \times 5)\end{array}$} & Autumn Royal & $100 \%$ & 0 & 0 & 0 & 0 \\
\hline & Crimson & $34.55 \%$ & $63.95 \%$ & $1.33 \%$ & $0.17 \%$ & 0 \\
\hline & Itum 4 & $1.02 \%$ & 0 & $98.98 \%$ & 0 & 0 \\
\hline & Itum5 & $15.69 \%$ & 0 & 0 & $84.31 \%$ & 0 \\
\hline & Itum9 & $10.26 \%$ & 0 & 0 & 0 & $89.74 \%$ \\
\hline \multirow{5}{*}{$\begin{array}{l}\text { F\#1(7×7×7) } \\
\text { F\#2(7×7×7) }\end{array}$} & Autumn Royal & $100 \%$ & 0 & 0 & 0 & 0 \\
\hline & Crimson & 0 & $100 \%$ & 0 & 0 & 0 \\
\hline & Itum4 & 0 & 0 & $100 \%$ & 0 & 0 \\
\hline & Itum5 & 0 & 0 & 0 & $100 \%$ & 0 \\
\hline & Itum9 & 0 & 0 & 0 & 0 & $100 \%$ \\
\hline
\end{tabular}

Table 7. Confusion matrix for the three configurations of the 3D convolution layer in the MBO architecture over test data.

\begin{tabular}{|c|c|c|c|c|c|c|}
\hline 3D Conv. & Variety & Autumn Royal & Crimson Seedless & Itum4 & Itum5 & Itum 9 \\
\hline \multirow{5}{*}{$\begin{array}{c}\text { F\#1(5 × } 5 \times 5) \\
\text { F\#2(10 × } 10 \times 10)\end{array}$} & Autumn Royal & $100 \%$ & 0 & 0 & 0 & 0 \\
\hline & Crimson & 0 & $100 \%$ & 0 & 0 & 0 \\
\hline & Itum4 & 0 & 0 & $100 \%$ & 0 & 0 \\
\hline & Itum5 & 0 & 0 & 0 & $100 \%$ & 0 \\
\hline & Itum9 & 0 & 0 & 0 & 0 & $100 \%$ \\
\hline \multirow{5}{*}{$\begin{array}{c}\text { F\#1(10 × } 10 \times 10) \\
\text { F\#2 }(5 \times 5 \times 5)\end{array}$} & Autumn Royal & $100 \%$ & 0 & 0 & 0 & 0 \\
\hline & Crimson & 0 & $100 \%$ & 0 & 0 & 0 \\
\hline & Itum4 & 0 & 0 & $100 \%$ & 0 & 0 \\
\hline & Itum5 & 0 & 0 & 0 & $100 \%$ & 0 \\
\hline & Itum9 & 0 & 0 & 0 & 0 & $100 \%$ \\
\hline \multirow{5}{*}{$\begin{array}{l}\mathrm{F} \# 1(7 \times 7 \times 7) \\
\mathrm{F} \# 2(7 \times 7 \times 7)\end{array}$} & Autumn Royal & $100 \%$ & 0 & 0 & 0 & 0 \\
\hline & Crimson & 0 & $100 \%$ & 0 & 0 & 0 \\
\hline & Itum4 & 0 & 0 & $100 \%$ & 0 & 0 \\
\hline & Itum5 & 0 & 0 & 0 & $100 \%$ & 0 \\
\hline & Itum9 & 0 & 0 & 0 & 0 & $100 \%$ \\
\hline
\end{tabular}

\section{Discussion}

Deep learning techniques require large numbers of images to create models that can generalise correctly. In the computer vision area, and orientated at tasks of object segmentation and identification, there are huge datasets labelled ImageNet [54] or LabelMe [20].

\subsection{Grape Classification}

In the field of plant biology, most repositories contain RGB datasets, but there is hardly any public HSI or MSI data available. For grape classification, datasets such as the one reported in [21] have been found, which contains 300 RGB images of five varieties, together with the coordinates of the position of 4398 bunches of grapes, or in [55], where there are 2078 RGB images of 15 grape varieties and where each image includes a reference marker for colorimetric analysis. The popular Kaggle website has a dataset of 1146 RGB images of grape grains.

In the field of HSI or MSI acquisition, no datasets related to grape varieties have been found; for this reason, a configurable multispectral vision system has been developed, which is able to capture images in the $400 \mathrm{~nm}-1000 \mathrm{~nm}$ range, as shown in Section 2.1. The system developed has made it possible to capture 1810 MSIs of five grape varieties 
and, using data augmentation techniques, have been transformed into 12,210 MSIs with 37 spectral bands. This set of images that is formed by 12,210 MSIs constitutes the first MSI grape grain dataset, and in future works, it will be expanded and made open-sourceavailable to the scientific community.

To evaluate the 3DeepM architecture with respect to other works, those that use deep learning techniques for grape classification have been selected. We found two common practices when using deep learning methods for classification: (1) use predefined architectures [2,56] (see Section 2.3.1) and (2) develop ad hoc architectures for a specific problem $[4,57,58]$.

These architectures have been designed to classify thousands of objects, and this enormous classification capacity entails a huge number of parameters in order for use. The final implementation of applications using these architectures require dedicated GPU-based platforms for their final use. In [2] the AlexNet architecture, transfer learning is used for the identification of six grape bunch varieties with an accuracy of 77.30\%. In [56], the authors present a classification model (ExtResnet) based on the extension of the Resnet architecture. The proposed architecture incorporates a block of FC layers to Resnet, together with a multiple-branch output. ExtResnet was used with 3967 images and obtained an accuracy of $99.92 \%$. The DeepGrapes model is presented in [57] and is composed of 2D convolution operations distributed in two blocks: a features extractor block and a classification block. The DeepGrapes architecture was trained to detect single white wine grapes with an average accuracy of $97.35 \%$. In [4], a custom architecture is presented that consists of four 2D convolutional layers with the ReLU function, each followed by a Max Pooling layer. This was trained with a set of 4564 samples consisting of six types of grape grains perfectly segmented and categorised by colour and obtained a $99.92 \%$ accuracy with a sample of 3967 images.

In the literature, there is only one case of multispectral grape measurement where deep learning techniques are used. In [58], RGB images captured with five different LED illuminations are used to classify the degree of maturity of grapes harvested during different weeks of the harvest period. The proposed architecture is similar to that used in [4] but with a lower number of parameters. The best result (accuracy $=93.41 \%$ ) was obtained in the classification of three ripening stages in a sample of 1260 grape grains.

Table 8 shows a summary of the grape classification methods based on deep learning techniques with which the 3DeepM architecture has been compared. The table shows the author, architecture type, image type, number of classes, the accuracy metric and the number of model parameters.

Table 8. Summary of the features of deep learning methods for grape classification.

\begin{tabular}{ccccccc}
\hline Author & $\begin{array}{c}\text { Architecture } \\
\text { Type }\end{array}$ & Image Type & $\begin{array}{c}\text { Total } \\
\text { Dataset }\end{array}$ & Classes & Accuracy & $\begin{array}{c}\text { Number of } \\
\text { Parameters }\end{array}$ \\
\hline$[2]$ & AlexNet & RGB & 201,824 & 6 & $77.30 \%$ & $62,379,752$ \\
{$[56]$} & Resnet & RGB & 3967 & 5 & $99.92 \%$. & $>25.6 \times 10^{6}$ \\
{$[57]$} & Ad hoc & RGB & 4000 & 1 & $97.35 \%$ & 293,426 \\
{$[4]$} & Ad hoc & RGB & 4565 & 6 & $100.00 \%$ & $3,459,275$ \\
{$[58]$} & Ad hoc & MSI & 1260 & 3 & $93.41 \%$ & 704,643 \\
3DeepM & Ad hoc & MSI & 12,210 & 5 & $100.00 \%$ & 25,565 \\
\hline
\end{tabular}

As can be observed in Table 7, the 3DeepM architecture is capable of correctly classifying $100 \%$ of the multispectral classes formed by 37 channels and is also 135 times lighter in terms of parameters than the other architecture that offers the best classification [4]. We believe that the capacity to obtain such a performance lies in two aspects: First the multispectral images used comprise a larger number of channels than a classic RGB, thus helping to obtain a dense dataset. Second these dense datasets are particularly amenable to DL processing thus obtaining extreme accuracy with fewer internal parameters. 


\subsection{Remote Sensing Applications}

The architectures reviewed in this work used in remote sensing applications have been specifically designed to be used with hyper- or multispectral images. In most cases [40-45,47-49], the authors apply the transfer learning technique, which means that they used a model that was not specifically trained for their case of study and adapted it via fine-tuning.

In this work, an architecture for multispectral image classification has been designed and implemented from scratch. Section 2.3.2 describes the design and implementation process carried out to obtain the 3DeepM architecture in detail. The high classification performance $(100 \%)$ obtained by 3DeepM is mainly due to two factors followed in the research process: (1) a specific multispectral vision system has been developed and an exhaustive sampling process has been carried out and (2) a systematic architecture design process was performed until an optimal design was obtained.

3DeepM can be used for multichannel image classification in remote sensing applications, as well as other types of applications, in two ways: (1) redesign the architecture using the detailed design and implementation steps shown in Section 2.3.2 or (2) use the transfer learning techniques described in the literature provided at the beginning of the section.

Finally, the exhaustive design process of 3DeepM has achieved an architecture with a very small number of parameters, which makes it suitable for online multispectral image classification applications on board autonomous robots or unmanned vehicles.

\section{Conclusions}

In this work, the design and implementation stages have been carried out for the 3DeepM architecture based on deep learning techniques, and the classification of multispectral images using this architecture has been performed. 3DeepM is characterised by having two 3D filter blocks specifically designed with 3D layers (3Dconv, 3DAvgPool and 3DGlobalAveragePool), which have allowed the spatial-spectral relationships of the different bands to be maximised in the images used for the validation. The article presents the optimisation procedures to determine the size and sequence of the kernels for the 3D convolutions that have made it possible to obtain $100 \%$ accuracy in the classification of multispectral images of five table grape varieties. The reduced number of 3D convolutions and the sequence have achieved a very light architecture in terms of the number of parameters, quickly trainable, and has also obtained the best results in comparison to other works in the literature.

The detailed design process described in this work for obtaining 3DeepM allows the use of the architecture in a multitude of applications that use multispectral images, such as remote sensing or medical diagnosis. In addition, the small number of parameters of 3DeepM make it ideal for application in online classification systems aboard autonomous robots or unmanned vehicles.

In future work, the dataset will be expanded with a greater number of samples and grape varieties, which will enable the validation of 3DeepM with a greater number of classes and a larger number of samples. In addition, the dataset will be published in open source for use by the scientific community. Finally, and due to the flexibility and reconfigurability of the multispectral computer vision system, work is being done to expand the research towards capturing multispectral images of other fruits and plant organs, such as leaves, flowers, etc.

Author Contributions: Conceptualisation, P.J.N., L.M., A.G.-N., D.J.A. and M.E.-C.; methodology, P.J.N., L.M., A.G.-N., M.V.D.-G. and M.E.-C.; software, P.J.N., L.M. and A.G.-N.; validation, P.J.N., L.M. and A.G.-N.; formal analysis, P.J.N., L.M., A.G.-N., and M.V.D.-G.; investigation, P.J.N., L.M., A.G.-N., M.V.D.-G. and M.E.-C.; resources, P.J.N., D.J.A. and M.E.-C.; data curation, P.J.N., L.M., A.G.-N., and M.V.D.-G.; writing - original draft preparation, P.J.N., L.M., A.G.-N., and M.E.-C.; writing-review and editing, P.J.N., L.M., A.G.-N., M.V.D.-G. and M.E.-C.; visualisation, P.J.N., L.M., A.G.-N., M.V.D.G., D.J.A. and M.E.-C.; supervision, P.J.N. and M.E.-C.; project administration, P.J.N. and M.E.-C.; 
funding acquisition, P.J.N. and M.E.-C. All authors have read and agreed to the published version of the manuscript.

Funding: This research was funded by BFU 2017-88300-C2-1-R to J.W. and M.E.-C, BFU 2017-88300C2-2-R to P.J.N. and CDTI 5117/17CTA-P to M.E.-C, P.J.N. and J.D.S.P.

Institutional Review Board Statement: Not applicable.

Informed Consent Statement: Not applicable.

Data Availability Statement: The dataset is available upon request from corresponding author.

Conflicts of Interest: The authors declare no conflict of interest.

\section{References}

1. Lowe, A.; Harrison, N.; French, A.P. Hyperspectral image analysis techniques for the detection and classification of the early onset of plant disease and stress. Plant Methods 2017, 13, 1-12. [CrossRef] [PubMed]

2. Pereira, C.S.; Morais, R.; Reis, M.J.C.S. Deep Learning Techniques for Grape Plant Species Identification in Natural Images. Sensors 2019, 19, 4850. [CrossRef]

3. Santos, T.T.; de Souza, L.L.; dos Santos, A.A.; Avila, S. Grape detection, segmentation, and tracking using deep neural networks and three-dimensional association. Comput. Electron. Agric. 2020, 170, 105247. [CrossRef]

4. Qasim El-Mashharawi, H.; Alshawwa, I.A.; Elkahlout, M. Classification of Grape Type Using Deep Learning. Int. J. Acad. Eng. Res. 2020, 3, 41-45.

5. Kamilaris, A.; Prenafeta-Boldú, F.X. Deep learning in agriculture: A survey. Comput. Electron. Agric. 2018, 147, 70-90. [CrossRef]

6. Hsieh, T.H.; Kiang, J.F. Comparison of CNN algorithms on hyperspectral image classification in agricultural lands. Sensors 2020, 20, 1734. [CrossRef]

7. Bhosle, K.; Musande, V. Evaluation of Deep Learning CNN Model for Land Use Land Cover Classification and Crop Identification Using Hyperspectral Remote Sensing Images. J. Indian Soc. Remote Sens. 2019, 47, 1949-1958. [CrossRef]

8. Xie, B.; Zhang, H.K.; Xue, J. Deep convolutional neural network for mapping smallholder agriculture using high spatial resolution satellite image. Sensors 2019, 19, 2398. [CrossRef]

9. Steinbrener, J.; Posch, K.; Leitner, R. Hyperspectral fruit and vegetable classification using convolutional neural networks. Comput. Electron. Agric. 2019, 162, 364-372. [CrossRef]

10. Kandpal, L.; Lee, J.; Bae, J.; Lohumi, S.; Cho, B.-K. Development of a Low-Cost Multi-Waveband LED Illumination Imaging Technique for Rapid Evaluation of Fresh Meat Quality. Appl. Sci. 2019, 9, 912. [CrossRef]

11. Li, B.; Cobo-Medina, M.; Lecourt, J.; Harrison, N.B.; Harrison, R.J.; Cross, J.V. Application of hyperspectral imaging for nondestructive measurement of plum quality attributes. Postharvest Biol. Technol. 2018, 141, 8-15. [CrossRef]

12. Veys, C.; Chatziavgerinos, F.; AlSuwaidi, A.; Hibbert, J.; Hansen, M.; Bernotas, G.; Smith, M.; Yin, H.; Rolfe, S.; Grieve, B. Multispectral imaging for presymptomatic analysis of light leaf spot in oilseed rape. Plant Methods 2019, 15, 4. [CrossRef]

13. Bravo, C.; Moshou, D.; West, J.; McCartney, A.; Ramon, H. Early disease detection in wheat fields using spectral reflectance. Biosyst. Eng. 2003, 84, 137-145. [CrossRef]

14. Yu, Y.; Liu, F. Dense connectivity based two-stream deep feature fusion framework for aerial scene classification. Remote Sens. 2018, 10, 1158. [CrossRef]

15. Perez-Sanz, F.; Navarro, P.J.; Egea-Cortines, M. Plant phenomics: An overview of image acquisition technologies and image data analysis algorithms. Gigascience 2017, 6, gix092. [CrossRef] [PubMed]

16. Paoletti, M.E.; Haut, J.M.; Plaza, J.; Plaza, A. Deep learning classifiers for hyperspectral imaging: A review. Isprs J. Photogramm. Remote Sens. 2019, 158, 279-317. [CrossRef]

17. Lacar, F.M.; Lewis, M.M.; Grierson, I.T. Use of hyperspectral imagery for mapping grape varieties in the Barossa Valley, South Australia. In Proceedings of the International Geoscience and Remote Sensing Symposium (IGARSS), Sydney, NSW, Autralia, 9-13 July 2001; Volume 6, pp. 2875-2877.

18. Knauer, U.; Matros, A.; Petrovic, T.; Zanker, T.; Scott, E.S.; Seiffert, U. Improved classification accuracy of powdery mildew infection levels of wine grapes by spatial-spectral analysis of hyperspectral images. Plant Methods 2017, 13, 47. [CrossRef]

19. Qiao, S.; Wang, Q.; Zhang, J.; Pei, Z. Detection and Classification of Early Decay on Blueberry Based on Improved Deep Residual 3D Convolutional Neural Network in Hyperspectral Images. Sci. Program. 2020, 4, 1-12. [CrossRef]

20. Russell, B.; Torralba, A.; Freeman, W.T. Labelme: The Open Annotation Tool. Available online: http:/ /labelme.csail.mit.edu/ Release3.0/browserTools/php/dataset.php (accessed on 30 December 2020).

21. Santos, T.; de Souza, L.; Andreza, d.S.; Avila, S. Embrapa Wine Grape Instance Segmentation Dataset-Embrapa WGISD. Available online: https: / / zenodo.org/record/3361736\#.YCx3LXm-thE (accessed on 17 January 2021).

22. Navarro, P.J.; Fernández, C.; Weiss, J.; Egea-Cortines, M. Development of a configurable growth chamber with a computer vision system to study circadian rhythm in plants. Sensors 2012, 12, 15356-15375. [CrossRef] 
23. Navarro, P.J.; Pérez Sanz, F.; Weiss, J.; Egea-Cortines, M. Machine learning for leaf segmentation in NIR images based on wavelet transform. In Proceedings of the II Simposio Nacional de Ingeniería Hortícola. Automatización y TICs en agricultura, Alemeria, Spain, 10-12 February 2016; p. 20.

24. Díaz-Galián, M.V.; Perez-Sanz, F.; Sanchez-Pagán, J.D.; Weiss, J.; Egea-Cortines, M.; Navarro, P.J. A proposed methodology to analyze plant growth and movement from phenomics data. Remote Sens. 2019, 11, 2839. [CrossRef]

25. Multispectral Camera MV1-D2048x1088-HS03-96-G2 । Photonfocus AG. Available online: https://www.photonfocus.com/ products / camerafinder/camera/mv1-d2048x1088-hs03-96-g2/ (accessed on 2 January 2021).

26. Multispectral Camera MV1-D2048x1088-HS02-96-G2 I Photonfocus AG. Available online: https://www.photonfocus.com/ products / camerafinder/camera/mv1-d2048x1088-hs02-96-g2/ (accessed on 2 January 2021).

27. Otsu, N. A Threshold Selection Method from Gray-Level Histograms. IEEE Trans. Syst. Man Cybern. 1979, 9, 62-66. [CrossRef]

28. Buslaev, A.; Iglovikov, V.I.; Khvedchenya, E.; Parinov, A.; Druzhinin, M.; Kalinin, A.A. Albumentations: Fast and flexible image augmentations. Information 2020, 11, 125. [CrossRef]

29. Goodfellow, I.; Bengio, Y.; Courville, A. Deep Learning; MIT Press: Cambridge, MA, USA, 2016.

30. Tran, D.; Bourdev, L.; Fergus, R.; Torresani, L.; Paluri, M. Learning spatiotemporal features with 3D convolutional networks. In Proceedings of the IEEE International Conference on Computer Vision, Santiago, Chile, 11-18 December 2015; Volume 2015, pp. 4489-4497.

31. Behnke, S. Hierarchical neural networks for image interpretation. Lect. Notes Comput. Sci. Incl. Subser. Lect. Notes Artif. Intell. Lect. Notes Bioinform. 2003, 2766, 1-220.

32. Yamaguchi, K.; Sakamoto, K.; Akabane, T.; Fujimoto, Y. A Neural Network for Speaker-Independent Isolated Word Recognition. In Proceedings of the First International Conference on Spoken Language Processing (ICSLP 90), Kobe, Japan, 18-22 November 1990.

33. LeCun, Y.; Bottou, L.; Bengio, Y.; Haffner, P. Gradient-based learning applied to document recognition. Proc. IEEE 1998, 86, 2278-2323. [CrossRef]

34. Krizhevsky, A.; Sutskever, I.; Hinton, G.E. ImageNet classification with deep convolutional neural networks. Commun. Acm 2017, 60, 84-90. [CrossRef]

35. Simonyan, K.; Zisserman, A. Very deep convolutional networks for large-scale image recognition. In Proceedings of the 3rd International Conference on Learning Representations, ICLR 2015-Conference Track Proceedings, San Diego, CA, USA, 7-9 May 2015.

36. Szegedy, C.; Liu, W.; Jia, Y.; Sermanet, P.; Reed, S.; Anguelov, D.; Erhan, D.; Vanhoucke, V.; Rabinovich, A. Going Deeper with Convolutions. In Proceedings of the IEEE Conference on Computer Vision and Pattern Recognition, Boston, MA, USA, 7-12 June 2015.

37. Szegedy, C.; Vanhoucke, V.; Ioffe, S.; Shlens, J. Rethinking the Inception Architecture for Computer Vision. In Proceedings of the IEEE Conference on Computer Vision and Pattern Recognition, Las Vegas, NV, USA, 27-30 June 2016; pp. $2818-2826$.

38. Szegedy, C.; Ioffe, S.; Vanhoucke, V.; Alemi, A. Inception-v4, Inception-ResNet and the Impact of Residual Connections on Learning. In Proceedings of the AAAI Conference on Artificial Intelligence, San Francisco, CA, USA, 4-9 February 2017.

39. Chollet, F. Xception: Deep Learning with Depthwise Separable Convolutions. In Proceedings of the IEEE Conference on Computer Vision and Pattern Recognition, Honolulu, HI, USA, 21-26 July 2017.

40. Hong, S.; Kim, S.; Joh, M.; Songy, S.K. Globenet: Convolutional neural networks for typhoon eye tracking from remote sensing imagery. arXiv 2017, arXiv:1708.03417.

41. Zhao, S.; Liu, X.; Ding, C.; Liu, S.; Wu, C.; Wu, L. Mapping Rice Paddies in Complex Landscapes with Convolutional Neural Networks and Phenological Metrics. GIScience Remote Sens. 2020, 57, 37-48. [CrossRef]

42. Zhou, Y.; Wang, M. Remote Sensing Image Classification Based on AlexNet Network Model. In Lecture Notes in Electrical Engineering; Springer: Berlin/Heidelberg, Germany, 2020; Volume 551, pp. 913-918.

43. Rezaee, M.; Mahdianpari, M.; Zhang, Y.; Salehi, B. Deep Convolutional Neural Network for Complex Wetland Classification Using Optical Remote Sensing Imagery. IEEE J. Sel. Top. Appl. Earth Obs. Remote Sens. 2018, 11, 3030-3039. [CrossRef]

44. Mahdianpari, M.; Salehi, B.; Rezaee, M.; Mohammadimanesh, F.; Zhang, Y. Very deep convolutional neural networks for complex land cover mapping using multispectral remote sensing imagery. Remote Sens. 2018, 10, 1119. [CrossRef]

45. Liu, K.; Yu, S.; Liu, S. An Improved InceptionV3 Network for Obscured Ship Classification in Remote Sensing Images. IEEE J. Sel. Top. Appl. Earth Obs. Remote Sens. 2020, 13, 4738-4747. [CrossRef]

46. Ma, H.; Liu, Y.; Ren, Y.; Wang, D.; Yu, L.; Yu, J. Improved CNN classification method for groups of buildings damaged by earthquake, based on high resolution remote sensing images. Remote Sens. 2020, 12, 260. [CrossRef]

47. Li, W.; Wang, Z.; Wang, Y.; Wu, J.; Wang, J.; Jia, Y.; Gui, G. Classification of High-Spatial-Resolution Remote Sensing Scenes Method Using Transfer Learning and Deep Convolutional Neural Network. IEEE J. Sel. Top. Appl. Earth Obs. Remote Sens. 2020, 13, 1986-1995. [CrossRef]

48. Jie, B.X.; Zulkifley, M.A.; Mohamed, N.A. Remote Sensing Approach to Oil Palm Plantations Detection Using Xception. In 2020 11th IEEE Control and System Graduate Research Colloquium, ICSGRC 2020-Proceedings; IEEE: Piscataway, NJ, USA, 2020 ; pp. 38-42.

49. Zhu, T.; Li, Y.; Ye, Q.; Huo, H.; Tao, F. Integrating saliency and ResNet for airport detection in large-size remote sensing images. In Proceedings of the 2017 2nd International Conference on Image, Vision and Computing, ICIVC 2017, Chengdu, China, 2-4 June 2017; pp. 20-25. 
50. Terry, M.I.; Ruiz-Hernández, V.; Águila, D.J.; Weiss, J.; Egea-Cortines, M. The Effect of Post-harvest Conditions in Narcissus sp. Cut Flowers Scent Profile. Front. Plant Sci. 2021, 11, 2144. [CrossRef] [PubMed]

51. de Castro, C.; Torres-Albero, C. Designer Grapes: The Socio-Technical Construction of the Seedless Table Grapes. A Case Study of Quality Control. Sociol. Rural. 2018, 58, 453-469. [CrossRef]

52. Royo, C.; Torres-Pérez, R.; Mauri, N.; Diestro, N.; Cabezas, J.A.; Marchal, C.; Lacombe, T.; Ibáñez, J.; Tornel, M.; Carreño, J.; et al. The major origin of seedless grapes is associated with a missense mutation in the MADS-box gene VviAGL11. Plant Physiol. 2018, 177, 1234-1253. [CrossRef] [PubMed]

53. Bradski, G. The OpenCV Library. Dr. Dobb's J. Softw. Tools 2000, 25, 120-125.

54. 54. Deng, J.; Dong, W.; Socher, R.; Li, L.-J.; Kai, L.; Li, F.-F. ImageNet: A large-scale hierarchical image database. In Institute of Electrical and Electronics Engineers (IEEE); IEEE: Piscataway, NJ, USA, 2010; pp. 248-255.

55. Seng, J.; Ang, K.; Schmidtke, L.; Rogiers, S. Grape Image Database-Charles Sturt University Research Output. Available online: https:/ / researchoutput.csu.edu.au/en/datasets/grape-image-database (accessed on 30 December 2020).

56. Franczyk, B.; Hernes, M.; Kozierkiewicz, A.; Kozina, A.; Pietranik, M.; Roemer, I.; Schieck, M. Deep learning for grape variety recognition. In Procedia Computer Science; Elsevier: Amsterdam, The Netherlands, 2020; Volume 176, pp. 1211-1220.

57. Śkrabánek, P. DeepGrapes: Precise Detection of Grapes in Low-resolution Images. Ifac Pap. 2018, 51, 185-189. [CrossRef]

58. Ramos, R.P.; Gomes, J.S.; Prates, R.M.; Simas Filho, E.F.; Teruel, B.J.; dos Santos Costa, D. Non-invasive setup for grape maturation classification using deep learning. J. Sci. Food Agric. 2020. [CrossRef] 